\title{
Parasitismo em Lagarta-do-Cartucho, Spodoptera frugiperda (Smith), na Região do Triângulo Mineiro, MG
}

\author{
Fábio M. A. Silva ${ }^{1}$, Harold G. Fowler ${ }^{2}$ e Raimunda N. S. Lemos ${ }^{3}$ \\ ${ }^{1}$ Departamento de Zoologia, IB/UNESP, Caixa postal 510, 18.618-900, Botucatu, SP. \\ ${ }^{2}$ Departamento de Ecologia, IB/UNESP, Caixa postal 199, 13.506-900, Rio Claro, SP. \\ ${ }^{3}$ Departamento de Fitotecnia e Fitossanidade, CCA/UEMA, Caixa postal 9, \\ 65.055-150, São Luis, MA.
}

An. Soc. Entomol. Brasil 26(2): 235-241 (1997)
Parasitism Associated with Fall Armyworm, Spodoptera frugiperda (Smith), in the Triangulo Mineiro Region, MG

\begin{abstract}
The frequency and distribution of parasitoids associated with fall armyworm larvae, Spodoptera frugiperda (Smith), was observed in commercial maize (Zea mays) fields, in the 1991/92 and 1992/93 seasons in the Triangulo Mineiro Region, MG, Brazil. Two regions in the 1991/92 season and five regions in the 1992/93 season were chosen. In each farm 100 larvae were collected in plants with 20 to 40 days after emergence. Larvae were reared in the laboratory at $25 \pm 2{ }^{\circ} \mathrm{C}, 50-60 \% \mathrm{RH}, 14 \mathrm{~h}$ - photoperiod and observed daily. The parasitoids more frequently observed were: Chelonus texanus (Cresson) (Hymenoptera: Braconidae), Diadegma sp. (Hymenoptera: Ichneumonidae), and Archytas incertus (Macquart) (Diptera: Tachinidae). The total parasitism ranged from $10,3 \%$ in $1991 / 92$ to $13,8 \%$ in $1992 / 93$. Dipteran parasitoids were more prevalent on fall armyworm pupae (76,5\%), in 1991/92 and 1992/93. In 1991/ 92 season the hymenopteran parasitoids were more prevalent on the 3rd and 4th instars larvae (41,9 and 45,2\%, respectively), and in 1992/93 on the 2 nd and 3rd instars larvae (39,1 and 43,5\%, respectively).
\end{abstract}

KEY WORDS: Insecta, Lepidoptera, Diptera, Hymenoptera, caterpillar, biological control.

RESUMO - Com o objetivo de avaliar a freqüência e distribuição de parasitóides associados a larvas da lagarta do cartucho, Spodoptera frugiperda (Smith), foram realizadas amostragens em campos comerciais da cultura do milho (Zea mays), nos anos agrícolas 1991/92 (duas regiões) e 1992/93 (cinco regiões). Em cada local amostrado foram coletadas aproximadamente 100 larvas, em plantas com 20 a 40 dias após a emergência. As larvas foram criadas em laboratório a $25 \pm$ $2^{\circ} \mathrm{C}$, UR $50-60 \%$, fotoperíodo de 14 horas e observadas diariamente. Os parasitóides mais frequientes foram: Chelonus texanus (Cresson) (Hymenoptera: Braconidae), Diadegma sp. (Hymenoptera: Ichneumonidae) e Archytas incertus (Macquart) (Diptera: Tachinidae). Foi observado que o parasitismo total foi de 10,3\% em 1991/92 e 13,8\% em 1992/93. Os parasitóides Diptera apresentaram preferência pela fase de pupa da lagarta do cartucho $(76,5 \%)$, nos dois anos agrícolas. Os parasitóides Hymenoptera tiveram preferência por lar 
vas de 3o e 4o ínstares (41,9 e 45,2\%), no ano agrícola 1991/92 e de 2o e 3oㅡㄴ ínstares (39,1 e 43,5\%), no ano agrícola 1992/93.

PALAVRAS-CHAVE: Insecta, Lepidoptera, Diptera, Hymenoptera, lagarta, controle biológico

Desfolhadora de importância econômica, com perdas estimadas na produção de $34 \%$, a lagarta-do-cartucho do milho, Spodoptera frugiperda (Smith), tem recebido atenção especial quanto ao desenvolvimento de métodos de controle que reduzam a aplicação de inseticidas. A atividade biológica dos inimigos naturais e da lagarta-do-cartucho tem sido pesquisada, mas suas interações pouco estudadas. Para isso é essencial que agentes de controle natural sejam identificados e seus efeitos sobre as populações de insetos-praga quantificados (Wall \& Berberet 1975). Vários exemplos de inimigos naturais adaptando-se a novos ambientes são relatados (Huffaker 1971). Portanto, o entendimento e conhecimento da ocorrência, identificação e abundância dos inimigos naturais são condições necessárias para se iniciar um programa integrado de controle (Henneberry et al. 1991). Entretanto, descrições detalhadas dos aspectos envolvidos nas interações predador-presa passam a ser fundamentais no sucesso do controle biológico.

Inimigos naturais, principalmente parasitóides, são encontrados em larvas de $S$. frugiperda, regulando a densidade do inseto (Barfield et al. 1980). Ashley (1979) relatou 53 espécies, de 43 gêneros e 19 famílias, parasitando larvas de $S$. frugiperda nas Américas, onde as famílias Braconidae, Ichneumonidae e Tachinidae ocorrem em 16, 19 e $47 \%$ dos gêneros, respectivamente. No Brasil, são descritas 12 espécies parasitando larvas de S. frugiperda (Luchini \& Almeida 1977, Patel \& Habib 1986, Valicente 1989), destacando-se os gêneros Campoletis, Chelonus e Archytas, sendo citados Campoletis flavicinta (Ashmead), Chelonus sp. e Archytas incertus (Macquart) (Patel \&
Habib 1986, Valicente 1989, Cruz et al. 1995). O objetivo deste trabalho foi relatar a freqüência, preferência e distribuição dos parasitóides nas diferentes fases de desenvolvimento larval de $S$. frugiperda, em cultivo comercial de milho, na região do triângulo mineiro, MG.

\section{Material e Métodos}

Locais Amostrados. Larvas de $S$. frugiperda foram coletadas em 12 campos de cultivo comercial de milho no ano agrícola 1991/92, nos municípios de Ituiutaba e Uberlândia e em 10 campos, no ano agrícola 1992/93, nos municípios de Araguari, Indianópolis, Ituiutaba, Uberaba e Uberlândia, MG, no período de dezembro até meados de fevereiro, durante os dois anos agrícolas. Em todos os locais amostrados, os cultivos próximos eram representados por soja (Glycine max), café (Coffea arabica) e matas.

Escolha da Àrea Amostrada. Em cada propriedade amostrada, foram coletadas 100 plantas com sintomas do ataque da praga. Foram amostradas 10 linhas de plantio, na parte central da área total e em cada linha foram avaliadas 10 plantas. Cada linha de plantio amostrada estava separada da outra por 10 linhas. As áreas amostradas não excediam a 10 ha e a idade da cultura estava em torno de 20 a 40 dias após a emergência. Todos os tratos culturais recomendados para a cultura foram efetuados, com exceção da aplicação de inseticidas. As plantas amostradas foram cortadas, acondicionadas em sacos plásticos e conduzidas ao laboratório. 
Criação em Laboratório. As larvas de $S$. frugiperda foram criadas em tubos de vidro (2,5cm de diâmetro x $8,5 \mathrm{~cm}$ de comprimento) fechados com algodão hidrófobo, à temperatura de $25 \pm 2{ }^{\circ} \mathrm{C}$, UR $50-60 \%$ e fotoperíodo de 14 horas, determinando-se os ínstares larvais pela medida da cápsula cefálica e a presença de posturas ou sinais externos de inimigos naturais. A seguir, as larvas foram incubadas individualmente e alimentadas com dieta artificial (Parra 1986). As larvas permaneceram no laboratório até a emergência dos adultos ou do parasitóide adulto, sendo as observações realizadas diariamente. Os parasitóides adultos foram colocados em álcool 70\%, etiquetados e identificados. As avaliações basearam-se na freqüência das espécies de parasitóides emergidos e distribuição da emergência dos parasitóides em cada ínstar do hospedeiro, localidade e período de coleta, comparados pelo teste de qui-quadrado $\left(\chi^{2}\right)$ (Sokal $\&$ Rohlf 1981).

\section{Resultados e Discussão}

Verificou-se, durante os dois anos agrícolas de levantamento, que os parasitóides

Tabela 1. Parasitóides emergidos de diferentes estádios de desenvolvimento larval e de pupas de $S$. frugiperda, nos municípios de Araguari, Indianópolis, Ituiutaba, Uberaba e Uberlândia, nos anos agrícolas 1991/92 e 1992/93.

\begin{tabular}{|c|c|c|c|}
\hline \multirow[t]{2}{*}{ Espécie de Parasitóide } & \multicolumn{2}{|c|}{ Ano Agrícola } & \multirow{2}{*}{$\begin{array}{c}\text { Estádio de Emergência } \\
\text { do Parasitóide }\end{array}$} \\
\hline & $91 / 92$ & $92 / 93$ & \\
\hline \multicolumn{4}{|l|}{ Hymenoptera } \\
\hline \multicolumn{4}{|l|}{ Braconidae } \\
\hline Macrocentrinae & & & \\
\hline $\begin{array}{l}\text { Macrocentrus sp. } \\
\text { Cheloninae }\end{array}$ & $02(01,75)^{1}$ & $00(00,00)^{1}$ & $3^{\circ}$ ínstar \\
\hline Chelonus texanus (Cresson) & $23(20,17)$ & $02(02,02)$ & $3^{\circ}, 4^{\circ}$ ínstar \\
\hline Microgastrinae & & & \\
\hline Cotesia sp. & $01(00,88)$ & $00(00,00)$ & $3^{\circ}$ ínstar \\
\hline Não identificado & $01(00,88)$ & $00(00,00)$ & $4^{\circ}$ ínstar \\
\hline \multicolumn{4}{|l|}{ Ichneumonidae: Porizontinae } \\
\hline Campoplex sp. & $01(00,88)$ & $00(00,00)$ & $3^{\circ}$ ínstar \\
\hline $\begin{array}{l}\text { Diadegma sp. } \\
\text { Ophioninae }\end{array}$ & $29(25,44)$ & $14(14,14)$ & $2^{\circ}, 3^{\circ}$ ínstares \\
\hline Ophion sp. & $02(01,75)$ & $01(01,01)$ & $5^{\circ}, 6^{\circ}$ ínstares \\
\hline Cremastinae & & & \\
\hline $\begin{array}{l}\text { Eiphosoma sp. } \\
\text { Gelinae }\end{array}$ & $02(01,75)$ & $06(06,06)$ & $4^{\circ}, 5^{\circ}$ ínstares \\
\hline Goryphina sp. & $01(00,88)$ & $00(00,00)$ & $3^{\circ}$ ínstar \\
\hline \multicolumn{4}{|l|}{ Diptera } \\
\hline Archytas incertus (Macquart) & $36(31,58)$ & $64(64,65)$ & pupa \\
\hline Lespesia affinis (Riley) & $14(12,28)$ & $04(04,04)$ & $5^{\circ}, 6^{\circ}$ ínstares \\
\hline Winthemia sp. & $01(00,88)$ & $08(08,08)$ & $5^{\circ}, 6^{\circ}$ ínstares \\
\hline Euphorocera sp. & $01(00,88)$ & $00(00,00)$ & pupa \\
\hline Total & 114 & 99 & \\
\hline
\end{tabular}

${ }^{1}$ Número entre parentesis indicam porcentagem de parasitismo, pelas espécies de parasitóides observadas em $S$. frugiperda, no conjunto dos locais amostrados. 
foram basicamente os mesmos, ocorrendo variação apenas na sua freqüência nos períodos amostrados. Treze espécies de parasitóides emergiram nas coletas realizadas no ano agrícola 1991/92, com quatro espécies representando $89,5 \%$ das espécies coletadas (Tabela 1). Os parasitóides Chelonus texanus (Cresson) e Diadegma sp. foram coletados de larvas de $3^{\circ}$ e $4^{\circ}$, e $2^{\circ}$ e e $3^{\circ}$ ínstares do hospedeiro, respectivamente, enquanto que Archytas incertus (Macquart) e Lespesia affinis (Riley) emergiram na fase de pupa, 5은 e 6o ínstares, respectivamente. Foram coletadas quatro espécies de Braconidae, cinco espécies de Ichneumonidae e quatro espécies de Tachinidae. Os dados (Tabela 1) indicam uma distribuição uniforme dos parasitóides com relação à fase de desenvolvimento do hospedeiro, indicando, assim, uma especialização dos himenópteros por fases iniciais de desenvolvimento e uma especialização dos dípteros por fases finais do hospedeiro, fato também constatados por Palomino (1965), Notz (1972), Patel et al. (1986) e Valicente (1989). Em todos os municípios avaliados, para os dois anos agrícolas estudados, foram coletadas 1106 larvas de $S$. frugiperda, emergindo 213 parasitóides $(19,3 \%)$, dos quais $11,4 \%$ não atingiram a fase adulta.

Quanto ao número de parasitóides emergidos nos anos agrícolas 1991/92 e 1992/ 93, para os municípios avaliados (Tabela 2), os níveis de parasitismo não variaram muito entre as localidades e de ano para ano. Entretanto, no município de Ituiutaba foi obtido um parasitismo de $41,7 \%$, quando analisado o número de larvas de $S$. frugiperda

Tabela 2. Mortalidade de S. frugiperda por parasitóides e causas desconhecidas, nos municípios de Araguari, Indianópolis, Ituiutaba, Uberaba e Uberlândia, nos anos agrícolas 1991/92 e 1992/93.

\begin{tabular}{|c|c|c|c|c|c|c|c|c|c|c|c|}
\hline \multicolumn{2}{|c|}{ Amostras } & \multicolumn{2}{|c|}{$\begin{array}{c}\text { Total de larvas } \\
\text { coletadas }\end{array}$} & \multicolumn{2}{|c|}{$\begin{array}{c}\text { Larvas } \\
\text { parasitadas(A) }\end{array}$} & \multicolumn{2}{|c|}{$\begin{array}{c}\text { Parasitóides } \\
\text { emergidos }(\mathrm{B})\end{array}$} & \multicolumn{2}{|c|}{$\begin{array}{c}\% \text { de parasitismo } \\
(\mathrm{A}+\mathrm{B})\end{array}$} & \multicolumn{2}{|c|}{$\begin{array}{c}\text { Mortes por causas } \\
\text { desconhecidas }\end{array}$} \\
\hline $91 / 92$ & $92 / 93$ & $91 / 92$ & $92 / 93$ & $91 / 92$ & $92 / 93$ & $91 / 92$ & $92 / 93$ & $91 / 92$ & $92 / 93$ & $91 / 92$ & $92 / 93$ \\
\hline $1^{1}$ & $1^{3}$ & 039 & 031 & 02 & 00 & 03 & 03 & 12,82 & 9,68 & 11 & 06 \\
\hline $2^{1}$ & $2^{3}$ & 097 & 041 & 02 & 05 & 11 & 06 & 13,40 & 26,83 & 05 & 15 \\
\hline $3^{1}$ & $3^{3}$ & 090 & 100 & 02 & 03 & 02 & 19 & 4,44 & 22,00 & 04 & 14 \\
\hline $4^{1}$ & $4^{4}$ & 093 & 100 & 09 & 03 & 10 & 18 & 20,43 & 21,00 & 25 & 09 \\
\hline $5^{1}$ & $5^{4}$ & 093 & 065 & 21 & 02 & 05 & 11 & 27,96 & 20,00 & 20 & 04 \\
\hline $6^{1}$ & $6^{4}$ & 096 & 095 & 27 & 01 & 13 & 18 & 41,67 & 20,00 & 34 & 06 \\
\hline $7^{1}$ & $7^{1}$ & 098 & 100 & 08 & 00 & 14 & 07 & 22,45 & 7,00 & 09 & 09 \\
\hline $8^{2}$ & $8^{5}$ & 100 & 075 & 04 & 01 & 07 & 06 & 11,00 & 9,33 & 13 & 14 \\
\hline $9^{2}$ & $9^{1}$ & 100 & 060 & 00 & 07 & 02 & 06 & 2,00 & 21,67 & 41 & 34 \\
\hline $10^{2}$ & $10^{2}$ & 100 & 100 & 13 & 00 & 14 & 06 & 27,00 & 6,00 & 13 & 44 \\
\hline $11^{2}$ & & 100 & & 08 & & 15 & & 23,00 & & 29 & \\
\hline $12^{2}$ & & 100 & & 05 & & 18 & & 23,00 & & 29 & \\
\hline
\end{tabular}

${ }^{1}$ Larvas coletadas em Ituiutaba - MG.

${ }^{2}$ Larvas coletadas em Uberlândia - MG.

${ }^{3}$ Larvas coletadas em Araguari - MG.

${ }^{4}$ Larvas coletadas em Indianópolis - MG.

${ }^{5}$ Larvas coletadas em Uberaba - MG. 
parasitadas e o número de parasitóides emergidos, para o ano agrícola 1991/92. Para a safra 1992/93, no município de Araguari safras 1991/92 e 1992/93 (Tabelas 3,4), observou-se uma relativa predominância dos parasitóides himenópteros no ano 1991/92.

Tabela 3. Freqüência de parasitóides emergidos em diferentes fases do desenvolvimento larval de $S$. frugiperda, em Ituiutaba e Uberlândia, no ano agrícola 1991/92.

\begin{tabular}{lccrrrr}
\hline $\begin{array}{l}\text { Fase de } \\
\text { desenvolvimento }\end{array}$ & \multicolumn{2}{c}{ Parasitóides/Diptera } & \multicolumn{2}{c}{ Hymenoptera } & \multicolumn{2}{c}{ Total } \\
\hline $\left.\mathrm{n}^{\circ}\right)$ & $(\%)$ & $\left(\mathrm{n}^{\circ}\right)$ & $(\%)$ & $\left.\mathrm{n}^{\circ}\right)$ & $(\%)$ \\
\hline $2^{\circ}$ instar & - & 0,00 & 04 & 3,51 & 04 & 3,51 \\
$3^{\circ}$ instar & - & 0,00 & 26 & 22,81 & 26 & 22,81 \\
$4^{\circ}$ instar & 03 & 2,63 & 28 & 24,56 & 31 & 27,19 \\
$5^{\circ}$ instar & 07 & 6,14 & 02 & 1,75 & 09 & 7,89 \\
$6^{\circ}$ instar & 06 & 5,27 & 02 & 1,75 & 08 & 7,02 \\
Pupa & 36 & 31,58 & - & 0,00 & 36 & 31,58 \\
\hline Total & 52 & 45,62 & 62 & 54,38 & 114 & 100,00 \\
\hline
\end{tabular}

$\chi^{2}=90,76-\mathrm{P}<0,0004$.

foram encontradas as maiores percentagens de parasitismo, com $26,8 \%$ e $22,0 \%$ de parasitóides emergidos e larvas parasitadas mortas. Quando analisadas as freqüências das distribuições da emergência com relação ao desenvolvimento larval de $S$. frugiperda, nas
Entretanto, para o ano 1992/93 houve alta predominância dos parasitóides dípteros.

Os resultados mostraram que a ocorrência dos parasitóides foi uniforme, na análise conjunta dos anos 1991/92 e 1992/93, verificando-se uma associação altamente

Tabela 4. Freqüência de parasitóides emergidos em diferentes fases do desenvolvimento larval de $S$. frugiperda, em Araguari, Indianópolis, Ituiutaba, Uberaba e Uberlândia, no ano agrícola 1992/93.

\begin{tabular}{lccrrrr}
\hline $\begin{array}{l}\text { Fase de } \\
\text { desenvolvimento }\end{array}$ & \multicolumn{2}{c}{ Parasitóides/Diptera } & \multicolumn{2}{c}{ Hymenoptera } & \multicolumn{2}{c}{ Total } \\
\hline $\left.\mathrm{n}^{\circ}\right)$ & $(\%)$ & $\left(\mathrm{n}^{\circ}\right)$ & $(\%)$ & $\left(\mathrm{n}^{\circ}\right)$ & $(\%)$ \\
\hline $2^{\circ}$ ínstar & - & 0,00 & 09 & 9,09 & 09 & 9,09 \\
$3^{\circ}$ instar & - & 0,00 & 10 & 10,10 & 10 & 10,10 \\
$4^{\circ}$ instar & - & 0,00 & 03 & 3,03 & 03 & 3,03 \\
$5^{\circ}$ instar & 08 & 8,08 & 01 & 1,01 & 09 & 9,09 \\
$6^{\circ}$ instar & 04 & 4,04 & - & 0,00 & 04 & 4,04 \\
Pupa & 64 & 64,65 & - & 0,00 & 64 & 64,65 \\
\hline Total & 76 & 76,77 & 23 & 23,23 & 99 & 100,00 \\
\hline
\end{tabular}

$\chi^{2}=94,02-\mathrm{P}<0,0004$. 
significativa $\left(\mathrm{c}^{2}=90,76, \mathrm{p}<0,0004\right.$ e $\mathrm{c}^{2}=$ 94,02, p < 0,0004) (Tabelas 3, 4), ou seja, nota-se que há uma diferença de parasitismo em relação ao hospedeiro, podendo-se dizer que ambas as ordens de parasitóides não competem pela mesma fase de desenvolvimento do hospedeiro, independente do município de coleta.

Nos anos agrícolas estudados, observa-se que a medida em que ocorre o desenvolvimento larval do hospedeiro, ocorre uma inversão da emergência dos parasitóides, havendo preferência por ínstares iniciais do hospedeiro pelos parasitóides Hymenoptera e por ínstares finais pelos parasitóides Diptera. De acordo com Notz (1972), os parasitóides Diptera possuem preferência pelos ínstares finais do hospedeiro devido a sua maior exigência alimentar, pois quando o parasitismo ocorre nos primeiros ínstares larvais, os dípteros não atingem a fase adulta, por não encontrarem alimento suficiente no hospedeiro. O mesmo não ocorre com himenópteros, que, segundo Palomino (1965), possuem exigências nutricionais diferentes.

Os dados apresentados nesse trabalho, mostram que as larvas de $S$. frugiperda foram parasitadas em diferentes localidades por um complexo de parasitóides, para os anos agrícolas 1991/92 e 1992/93, verificando-se que existe uma dependência direta do número de parasitóides emergidos por número de larvas coletadas, ou seja, uma regulagem dependente da densidade populacional (ocorrendo aumento da população de parasitóides, quando ocorre aumento da população da praga). Através das análises realizadas neste trabalho, os inimigos naturais encontrados constituem-se em importantes componentes na regulação da população de S. frugiperda.

\section{Agradecimentos}

Ao Dr. José H. Guimarães do ICB/USP, pela identificação dos parasitóides Diptera (Tachinidae), à Dra. Angélica M. PenteadoDias da UFSCar pela identificação dos parasitóides Hymenoptera (Braconidae e
Ichneumonidae) e à FAPESP, pela bolsa concedida (processo n ${ }^{\circ}$ 91/1720-6).

\section{Literatura Citada}

Ashley, T.R. 1979. Classification and distribution of fall armyworm parasites. Fla. Entomol. 62: 114-123.

Barfield, C.S., J.L. Stimac \& M.A. Keller. 1980. State-of-the-art for predicting damaging infestations of fall armyworm. Fla. Entomol. 63: 364-375.

Cruz, I., D.A.N. Lima, M.L.C. Figueiredo \& F.H. Valicente. 1995. Aspectos biológicos do parasitóide Campoletis flavicinta (Ashmead) criado em lagartas de Spodoptera frugiperda (Smith). An. Soc. Entomol. Brasil 24:201-208.

Henneberry, T.J., P.V. Vail, A.C. Pearson \& V. Sevacherian. 1991. Biological control agents of noctuid larvae (Lepidoptera: Noctuidae) in the Imperial Valley of California. Southwest. Entomol. 16: 81-89.

Huffaker, C.B. 1971. Biological control. New York, Plenum, 511 p.

Lucchini, F. \& A.A. Almeida. 1980. Parasitas da Spodoptera frugiperda (Smith \& Abbot, 1797) (LepidopteraNoctuidae), lagarta do cartucho do milho, encontrados em Ponta Grossa-Pr. An. Soc. Entomol. Brasil. 9: 115-121.

Notz, P.A. 1972. Parasitismo de Diptera e Hymenoptera sobre larvas de Spodoptera frugiperda (Smith) (LepidopteraNoctuidae) recolectadas em maiz, Maracay, Venezuela. Rev. Fac. Agronom. Venezuel. 6: 5-16.

Parra, J.R.P. 1986. Criação de insetos para estudo com patógenos, p.348-373. In S.B. 
Alves (ed.), Controle microbiano de insetos. São Paulo, Manole, 407 p.

Patel, P.N. \& M.E.M. Habib. 1986. Levantamento e eficiência de insetos parasitos de Spodoptera frugiperda (Smith \& Abbot, 1797) (LepidopteraNoctuidae). Rev. Agric. 61:93-100.

Sokal, R.R. \& F.J. Rohlf. 1981. Biometry. New York, Freeman \& Company, 859 p.

Valicente, F.H. 1989. Levantamento dos inimigos naturais de Spodoptera frugiperda (J.E. Smith, 1797) (Lepidoptera-Noctuidae) em diferentes regiões do estado de Minas Gerais. An. Soc. Entomol. Brasil 18: 119-130.

Wall, R. \& R.C. Berberet. 1975. Parasitoids associated with lepidopterous pests on peanuts, Oklahoma fauna. Environ. Entomol. 11: 877-882.

Recebido em 28/08/96. Aceito em 19/06/97. 